\title{
AF and Diabetes Prognosis and Predictors
}

\author{
Carlo Pappone, Francesca Zuffada and Vincenzo Santinelli \\ Department of Arrhythmology, Maria Cecilia Hospital, Cotignola, Ravenna, \\ Italy
}

\section{Introduction}

Although approximately $10 \%$ of atrial fibrillation (AF) patients have no evident cardiac disorder (so-called "lone" AF), the arrhythmia usually occurs in patients with structural heart disease. Hypertension, coronary heart disease, valvular heart disease, dilated cardiomyopathy, and heart failure are the most frequent cardiovascular comorbidities associated with AF, but this arrhythmia is frequently found also in diabetic patients, with prevalence rates estimated to be at least double than among patients without diabetes (1). Comorbidities may induce atrial fibrosis and loss of myocardial tissue which have an apparent clear impact in facilitating AF by reducing the conduction velocity and possibly creating areas of conduction block. Fibrosis may be either a substrate for AF or a result of fibrillating atria and part of the so-called structural remodeling. Since atrial enlargement is often present in AF patients with or without comorbidities, it is difficult to establish if it represents the cause or the consequence of the arrhythmia. AF affects one in 25 adults aged 60 or over and nearly one in 10 adults aged 80 or over. Due to symptoms and the increased risk of ischemic stroke and death in elderly patients, AF is a source of considerable concern, and its impact is likely to increase as the number of individuals affected by AF rises nearly 2.5-fold during the next 50 years. The economic repercussions on national health systems around the world will be considerable. Like AF, diabetes mellitus is a global health problem with an estimated worldwide prevalence of $2.8 \%$ in 2000, which will increase to $4.4 \%$ in 2030 (2). At least 10.3 million Americans carry a diagnosis of diabetes mellitus. Another 5.4 million of people are estimated to have undiagnosed diabetes. Approximately $90 \%$ of patients with diabetes have the type 2 variety. The onset of type 2 diabetes usually precedes clinical diagnosis by several years. As a result, an increasing prevalence of type 2 diabetes cannot be divorced from the rising prevalence of atrial fibrillation in our society. Diabetic patients have at least twice the risk of vascular complications and cardiovascular death, compared with those without diabetes(3,4). Several epidemiological and pathological data have reported that diabetes is an independent predictor of cardiovascular disease (CVD) in both men and women. CVDs are considered as the cause of death in $\approx 65 \%$ of people with diabetes. To make matters worse, when patients with diabetes develop clinical CVD, they sustain a worse prognosis for survival than do CVD patients without diabetes. These observations suggest that it is imperative to promote coordinated efforts on behalf of cardiologistis, electrophysiologistis, neurologists, and primary-care providers to meet the 
increasing challenge of stroke and death prevention and rhythm management in the growing population of patients with atrial fibrillation and diabetes. Since among patients with AF type 2 diabetes is frequently associated with cardiovascular comorbidities including coronary artery disease, heart failure and hypertension (AF has been observed three times higher in patients with hypertension)(5) it is very difficult to evaluate its independent predictive value for arrhythmia progression, thromboembolic and cardiac adverse events including death $(6,7)$. To assess whether diabetes further increases the already high risk among patients with $\mathrm{AF}$ and concomitant cardiovascular diseases, a prospective large number of patients with a long-term follow-up is required. We report here our long-term experience in a large and selected population of patients with paroxysmal AF in order to identify predictors of outcome, including AF progression, ischemic stroke and cardiovascular death.

\section{Study population}

Patients with history of documented paroxysmal AF with at least 2 or more episodes of symptomatic or asymptomatic AF lasting $>1$ hour were recruited for this 4 -year prospective follow-up study. Exclusion and inclusion criteria are reported in Table 1. Patients with arrhythmia due to potentially reversible causes such as acute or recent ( $<6$ months) myocardial infarction, recent cardiac surgery, New York Heart Association class III-IV heart failure, severe valvular heart disease requiring surgery, uncontrolled hypertension, acute pulmonary disease, WPW syndrome, a history of long-QT syndrome, Brugada syndrome, pericarditis, substance abuse, electrolyte imbalance, hyper or hypothyroidism were excluded. Patients with hepatic diseases, history of thromboembolism including stroke or TIA, contraindication to anticoagulation therapy, or any condition that would make survival for 1 year unlikely were also excluded. Patients scheduled to undergo catheter ablation or implantation of a pacemaker or defibrillator were also excluded. All participants had to have been on stable therapy for AF and any underlying cardiovascular disorders for at least 1 month before enrollment. Patients were allowed to continue all previously prescribed treatments for these conditions (including antiarrhythmic drugs, amiodarone, beta-blockers, and ACE inhibitors).

\subsection{Follow-up}

Serial control visits with ECG, 48-h Holter, echocardiography, and laboratory testing were scheduled at 1, 3, 6 months and thereafter once annually for 4 years. If a recurrence of $\mathrm{AF}$ was detected, the patient was asked to come in for an office visit to confirm the findings. Baseline evaluation included a past medical history of cardiovascular and endocrine disorders as well as a history of precipitating events. A complete blood count, urinalysis, thyroid-function tests, hepatic panel, lipid profile, and serum chemical measurements were obtained at baseline and at each control visit thereafter. Transesophageal echocardiography was performed at baseline to rule out atrial thrombi and annually. Recurrent episodes were pharmacologically managed by conventional ADT (propafenone, flecainide, and/or sotalol as first-line drugs in patients without structural heart disease or amiodarone as a single drug or in combination in patients with structural heart disease or in case of first-line drugs failure) according to AF management guidelines $(8,9)$. Electrical cardioversion was performed if necessary or in patients with persistent AF refractory to ADT. Antithrombotic 
therapy with warfarin (INR between 2.0 and 3.0) was initially applied after stroke risk assessment according to the current AF guidelines recommendations $(8,9)$. Patients with comorbidities or those with at least one moderate risk factor for thromboembolism regardless of AF clinical form were prescribed warfarin. Electrical cardioversion was performed for a maximum of 3 attempts. Patients with recurrent AF were followed monthly in an outpatient setting. The outcomes evaluated in this analysis were arrhythmia progression, cardiovascular mortality and major thromboembolic events.

Inclusion criteria:

Age between 18 and 85

\section{Exclusion criteria:}

Left ventricular ejection fraction $<30 \%$

NYHA functional class III-IV

Left atrial size $>60 \mathrm{~mm}$

AF burden $>2$ episodes/month

Uncorrected severe valvular heart disease

Contraindication to anticoagulation

Presence of left atrial thrombus

Patients scheduled to undergo catheter ablation or implantation of a pacemaker or defibrillator

Prior attempt at catheter or surgical ablation for atrial fibrillation

Recent (6 months) history of myocardial infarction

Cardiac surgery for congenital, valvular, aortic or coronary heart disease

History of cerebrovascular accidents

Pregnancy

Life expectancy less than 1 year

Significant comorbidities: cancer, end stage renal disease, severe obstructive lung disease, cirrhosis, etc

Inability or unwillingness to provide written informed consent

Table 1. Inclusion and Exclusion Criteria

\subsection{Definitions}

Paroxysmal AF was defined as recurrent AF that was self-terminating within 7 days. Persistent AF is defined as AF that was sustained beyond 7 days, or lasting less than 7 days but requiring pharmacologic or electrical cardioversion for termination. Ischemic stroke was defined as neurological deficit of sudden onset lasting $>24$ hours and caused by ischemia. Cardiovascular (CV) death was defined as death due to cardiovascular causes (myocardial infarction or heart failure).

\section{Results}

\subsection{Study population}

Among 5210 screened patients with paroxysmal AF, 1931 (mean age, 61.5 years, M, 47.2\%) fulfilled inclusion and exclusion criteria and completed a 4-year follow-up period. Baseline clinical characteristics of the study population are shown in Table 1. Many patients had a 
history of hypertension with hypetensive heart disease $(44.8 \%)$ or coronary artery disease $(31.6 \%)$. A total of $9.5 \%$ of patients had recent heart failure and $8.8 \%$ had valvular heart disease often with mitral valve disease. Type 2 diabetes mellitus was present in $13.9 \%$ of patients and in many of them (85 patients) diabetes was of long duration (median duration 15 years) and poor controlled with oral antidiabetic therapy as assessed by glycosylated hemoglobin (median 8\%)). AF burden was $2.05 \pm 0.2$ (episodes/month). At the time of enrollment, $35 \%$ of the patients were receiving amiodarone alone or in combination, $25.2 \%$ were receiving beta-blockers or sotalol, $20 \%$ were receiving ACE inhibitors, and only $20 \%$ of them were receiving chronic anticoagulant therapy with warfarin. Class I antiarrhythmic agents were prescribed in $30 \%$ of patients. Adverse drug reactions occurred in 115 patients during class I antiarrhythmic drugs (75 patients), amiodarone alone (30 patients) or in combination (10 patients). No serious complications developed and long-term antiarrhythmic drug therapy was not permanently discontinued in any patient.

\subsection{Atrial fibrillation progression}

At 4 years, 1452 patients had had at least a recurrence of AF , 183 patients remained in stable sinus rhythm on antiarrhythic drug therapy and the remaining 276 patients had progressed to persistent AF despite conventional ADT alone or in combination. Intensive Holter recordings revealed that in 68/183 patients, of whom 50 with diabetes mellitus, AF progression was silent. The baseline characteristics of patients with and without AF progression were compared for demographics at study entry (Table 2). Patients who progressed were older and had more comorbidities including type 2 diabetes and higher baseline AF burden than those who did not. On the baseline echocardiogram, patients with AF progression had larger left atrial diameters. The median interval between the onset of recurrent paroxysmal and persistent AF was 33.5 months (range 5-38). Patients who progressed received more frequently drugs associated with heart failure (ACE inhibitors and/or diuretics) and many of them had oral anticoagultion therapy with warfarin. Electrical cardioversion was more frequently used before and after progression but the arrhythmia recurred $32 \pm 18$ days after electrical cardioversion requiring multiple hospitalizations.

\subsection{Complications}

Cardiovascular events. During a median follow-up of 35 months, 84 patients died due to cardiovascular causes (Table 2) with a cumulative 4 -year cardiovascular mortality rate of $4.3 \%$. All patients were older than 65 years of age and all had comorbidities including heart failure, diabetes, coronary artery disease and hypertensive cardiomyopathy alone or in combination. Arrhythmia progression occurred in $49 / 84$ (58.3\%) before death which was commonly due to worsening of heart failure or acute heart failure (55 patients) secondary to inadequate control of the ventricular rate at the time of recurrent episodes of persistent arrhythmia at rapid ventricular rate $(115 \pm 15 \mathrm{bpm})$ or hypertensive crises with pulmonary edema ( 29 patients). All patients at the time of death were on amiodarone therapy and warfarin. Thromboembolic events. During a median follow-up of 25 months, thromboembolism developed in 140 patients (Table 2) with a 4-year cumulative stroke rate of $7.3 \%$. Arrhythmia progression occurred in 58/140 (41.4\%) before thromboembolic events. All patients at the time of thromboembolism were on warfarin but subtherapeutic doses were found in many of them $(40 \%)$. 


\begin{tabular}{|c|c|c|c|c|}
\hline Variables & $\begin{array}{l}\text { All Patients } \\
(\mathrm{n}=1931)\end{array}$ & $\begin{array}{l}\text { No AF Progression } \\
(n=1655)\end{array}$ & $\begin{array}{l}\text { AF Progression } \\
(\mathrm{n}=276)\end{array}$ & p-value \\
\hline Male sex, $\mathrm{n}(\%)$ & $911(47.2)$ & 1095(66.1) & 165 (59.7) & 0.024 \\
\hline Age, (years) & $61.5 \pm 7.4$ & $61.2 \pm 7.4$ & $62.4 \pm 7.2$ & 0.021 \\
\hline Hypertension, n (\%) & $865(44.8)$ & $718(43.8)$ & $147(53.2)$ & 0.001 \\
\hline Heart Failure, n (\%) & $184(9.5)$ & $119(7.1)$ & $65(23.5)$ & $<0.001$ \\
\hline Diabetes mellitus, n (\%) & $270(13.9)$ & $188(11.3)$ & $82(29.7)$ & $<0.001$ \\
\hline Valvular disease, $\mathrm{n}(\%)$ & $171(8.8)$ & $141(8.5)$ & $35(12.6)$ & 0.020 \\
\hline Coronary artery disease & $611(31.6)$ & $514(31)$ & $93(33.6)$ & 0.382 \\
\hline LA diameter, $(\mathrm{mm})$ & $42 \pm 3.3$ & $41.5 \pm 3.2$ & $42.8 \pm 3.4$ & $<0.001$ \\
\hline LV EF, (\%) & $54 \pm 5.3$ & $54 \pm 5.0$ & $51 \pm 6.5$ & $<0.001$ \\
\hline $\begin{array}{l}\text { AF burden, } \\
\text { (episodes/month) }\end{array}$ & $2.2 \pm 0.5$ & $2.2 \pm 0.4$ & $2.4 \pm 0.7$ & $<0.001$ \\
\hline AF duration, (months) & $50.4 \pm 7.3$ & $53 \pm 3.5$ & $58 \pm 4.6$ & $<0.001$ \\
\hline Thromboembolism, n (\%) & $140(7.2)$ & $82(4.9)$ & $58(21)$ & $<0.001$ \\
\hline CV Death, $\mathrm{n}(\%)$ & $84(4.3)$ & $36(2.1)$ & $48(17.3)$ & $<0.001$ \\
\hline
\end{tabular}

Table 2. Baseline characteristics of patients with paroxysmal AF

\subsection{Risk factors of progression, thromboembolic events and death}

Multivariate analysis demonstrated that many comorbidities including diabetes mellitus, heart failure, and coronary artery disease were common predictors of arrhythmia progression as well as of thromboembolic events and death (Tables 3-5). In addition, low ejection fraction, left atrial enlargment, female gender, and older age also predicted all adverse events. AF burden was a strong predictor of arrhythmia progression but did not predict thromboembolic events/death. Diabetes and heart failure were the strongest predictors of all events. (Tables 3-5).

\begin{tabular}{llcccc}
\hline Covariates & $\begin{array}{l}\text { Regression } \\
\text { Coefficient }\end{array}$ & p-value & Adjusted HR & \multicolumn{2}{c}{ 95\% CI } \\
\hline AF burden & 0.545 & $<0.001$ & 1.724 & 1.449 & 2.051 \\
Ejection fraction & -0.070 & $<0.001$ & 0.933 & 0.912 & 0.954 \\
Sex & 0.335 & 0.031 & 1.398 & 1.032 & 1.894 \\
Age & 0.043 & 0.001 & 1.044 & 1.018 & 1.070 \\
Coronary artery disease & 0.328 & 0.015 & 1.388 & 1.067 & 1.806 \\
Heart failure & 0.674 & $<0.001$ & 1.963 & 1.412 & 2.728 \\
Diabetes mellitus & 0.568 & $<0.001$ & 1.765 & 1.301 & 2.396 \\
Left atrial diameter & 0.050 & 0.007 & 1.051 & 1.013 & 1.090 \\
Valvular disease & 0.537 & 0.004 & 1.712 & 1.191 & 2.460 \\
\hline
\end{tabular}

Table 3. Multivariate Cox analysis for AF progression

\section{Discussion}

This is the largest prospective long-term study which provides additional data on predictors of arrhythmia progression and prognosis among a selected population with paroxysmal AF 
which represents the vast majority of patients with paroxysmal AF commonly seen in clinical practice. The long-term results while confirming the role of associated comorbidities to predict AF progression, clearly demonstrate that type 2 diabetes is one of the strongest predictor of both arrhythmia progression and prognosis. AF burden of at least 2 episodes/month was a strong predictor of arrhythmia progression but this arrhythmia burden, unlike diabetes and cardiovascular comorbidities did not predict stroke and death. AF progression was discrete and at 4 years occurred only in a minority of patients $(14.3 \%)$, most of whom with advancing age and comorbidities.

\begin{tabular}{lllccc}
\hline Covariates & $\begin{array}{l}\text { Regression } \\
\text { Coefficient }\end{array}$ & p-value & $\begin{array}{c}\text { Adjusted } \\
\text { HR }\end{array}$ & \multicolumn{2}{c}{ 95\% CI } \\
\hline Ejection fraction & -0.043 & 0.005 & 0.958 & 0.930 & 0.987 \\
Sex & 0.696 & 0.004 & 2.005 & 1.256 & 3.202 \\
Age & 0.120 & $<0.001$ & 1.128 & 1.082 & 1.176 \\
Coronary artery disease & 1.345 & $<0.001$ & 3.839 & 2.207 & 6.678 \\
Heart failure & 1.407 & $<0.001$ & 4.085 & 2.533 & 6.587 \\
Diabetes mellitus & 1.705 & $<0.001$ & 5.500 & 3.208 & 9.431 \\
Left atrial diameter & 0.102 & $<0.001$ & 1.108 & 1.056 & 1.162 \\
\hline
\end{tabular}

Table 4. Multivariate Cox analysis for thromboembolism

\begin{tabular}{lllccc}
\hline Covariates & $\begin{array}{l}\text { Regression } \\
\text { Coefficient }\end{array}$ & p-value & $\begin{array}{c}\text { Adjusted } \\
\text { HR }\end{array}$ & \multicolumn{2}{c}{ 95\% CI } \\
\hline Ejection fraction & -0.066 & $<0.001$ & 0.936 & 0.904 & 0.970 \\
Sex & 1.576 & $<0.001$ & 4.836 & 2.017 & 11.594 \\
Age & 0.057 & 0.054 & 1.059 & 0.999 & 1.121 \\
Coronary artery disease & 0.741 & 0.051 & 2.097 & 0.996 & 4.417 \\
Heart failure & 2.557 & $<0.001$ & 12.891 & 4.977 & 33.387 \\
Diabetes mellitus & 1.628 & $<0.001$ & 5,092 & 2,233 & 11.615 \\
Left atrial diameter & 0.059 & 0.065 & 1.061 & 0.996 & 1.130 \\
\hline
\end{tabular}

Table 5. Multivariate Cox analysis for cardiovascular death

\subsection{Predictors of AF progression, ischemic stroke and death}

Risk factors for the transition from paroxysmal to persistent forms of AF may be similar to those predisponing to incident $\mathrm{AF}$, but there are very limited data on their identification for secondary prevention in order to reduce arrhythmia progression and associated complications such as thromboembolic events or death. Patients with AF have a substantial risk of stroke, which is modified by the presence or absence of several risk factors. These risk factors have been used to develop thromboembolic risk stratification schemes, which have arbitrarily divided the risk into low, intermediate, and high risk strata. Multivariate pooled analysis demonstrated that cardiovascular comorbidities and diabetes were independently predictive of arrhythmia progression as well as of stroke and CV death while baseline AF burden of 2 episodes/month was a strong predictor of arrhythmia progression, but at 4 years was not sufficient to predict serious adverse events and death. Our data also demonstrate that advancing age, female gender and the presence of coronary artery disease 
are independently predictive of adverse events including death. These findings suggest that in patients with paroxysmal AF thromboembolic risk stratification schemes using these risk factors are useful. Therefore, potential additional risk factors listed in the 2006 ACC/AHA/ESC guidelines (8) as "less validated or weaker risk factors" including female gender, age 65-74 years, and coronary artery disease should be instead considered as independent strong risk factors. Many of these variables have been expressed in the CHA2DS2-VASc (Congestive heart failure, Hypertension, Age $>75$ years, Diabetes mellitus, previous Stroke/transient ischemic attack, Vascular disease, Age 65-74 years, Sex category,) score, which has been proposed to complement the CHADS2 score.

\subsection{Silent progression of AF}

In the present study, AF progression from paroxysmal to persistent AF was silent in many patients as documented by the intensive Holter monitoring. Of note, about a half of patients with AF progression had diabetes mellitus. These findings are important in terms of pathophysiology and therapy suggesting that oral anticoagulation therapy with warfarin in patients at high risk should be frequently and constantly monitored to avoid subtherapeutic intensity (INR below 2.0) regardless of the presence of symptoms. In the absence of an accurate arrhythmia monitoring by intermittent ECG recordings, asymptomatic transition to more persistent forms, particularly in diabetic patients, may be undetected increasing the risk of theromboembolic and cardiovascular complications.

\subsection{Comparison with previous studies}

The results of the present study extend the results of previous studies on AF progression from paroxysmal to persistent AF (10-14). In the CARAF (Canadian Registry of Atrial Fibrillation Study) study, underlying heart disease and age were reported as independent factors of AF progression (10). Nieuwlaat et al reported univariate analysis of AF progression in the Euro Heart Survey (EHS), but independent risk factors were not analyzed (11). De Vos et al (12) have recently reported interesting data on the clinical correlates of AF progression and prognosis in the patients enrolled in the Euro Heart Survey of AF (13). In agreement with our data but during a much shorter follow-up period (only 1 year), progression to persistent AF occurred in 15\% of Euro Heart Survey patients. Based on these data, the Authors have suggested a new risk stratification score (HATCH) to identify patients prone to arrhythmia progression. Recently, we have reported AF progression in 106 patients with first of whom 52 with comorbidities $\mathrm{AF}$ and diabetes was a strong predictor of arrhythmia progression (14). The present study reports additional data among a large patient population with paroxysmal AF. A predefined baseline AF burden before enrollment was purposively included to enhance the clinical applicability of the results and, unlike the study by de Vos et al (12), patients with AF who required pharmacological cardioversion for restoration of sinus rhythm were considered as persistent AF patients and then were excluded. The results at 4 years while confirming the independent role of many cardiovascular comorbidities as predictors of AF progression, for the first time show type 2 diabetes mellitus as a strong independent predictor of AF progression, thromboembolism and cardiovascular death. In the analysis of de Vos and colleagues (12), diabetes also occurred more frequently in patients who progressed as compared with those who did not (19\% vs $14 \%$ ), but no significant differences were found and this may be due to several reasons including a shorter follow-up, misclassification of paroxysmal AF in many patients 
and a less accurate rhythm monitoring. Analysis of AF progression and predictors of prognosis requires a well defined AF burden prior to enrollment which should not be based on the patients'reported history alone as it may be unreliable. Previous trials frequently have been based exclusively on electrocardiography performed at scheduled visits or on the patient's report of symptoms which may explain why different rates of progression and predictors have been reported. Recently, the results of the ADVANCE study suggested that $\mathrm{AF}$ in diabetic patients should be regarded as a marker of adverse outcome and prompt aggressive management of all risk factors (15). Although it seems to be conceivable that many comorbidities including type 2 diabetes should be independent predictors of stroke and mortality in patients with nonvalvular atrial fibrillation, available data do not provide strong support. The results of the present study demonstrate that type 2 diabetes as well as cardiovascular comorbidities are independently predictors of thromboembolism and cardiovascular death. We believe that patients with paroxysmal AF without prior stroke with at least one risk factor such as advancing age, underlying heart or metabolic disease and a well defined baseline arrhythmia burden, as those enrolled in the present study, indeed represent the vast majority of patients with AF commonly seen in real life clinical practice. Large clinical studies require years for completion, but they are required to determine the impact of several risk factors on mortality and other long-term outcomes. In our experience, older patients, particularly females with cardiovascular comorbidity and diabetes mellitus are at highest risk of adverse outcomes and require chronic anticoagulation therapy with warfarin.

\section{Conclusions}

Our experience provides new evidence on characteristics and outcome among a large cohort of selected patients with paroxysmal AF with a minimal use of anticoagulation treatment. These data, while confirming the role of many cardiovascular comorbidities in predicting adverse outcomes, demonstrate that diabetes mellitus is an independent strong predictor of $\mathrm{AF}$ progression, stroke and $\mathrm{CV}$ death. AF progression in diabetics may be frequently silent which may result in higher risk of adverse outcome.

\section{References}

[1] Movahed MR, Hashemzadeh M, Jamal MM. Diabetes mellitus is a strong, independent risk for atrial fibrillation and flutter in addition to other cardiovascular disease. Int J Cardiol 2005;105:315-318.

[2] Wild S, Roglic G, Green A, Sicree R, King H. Global prevalence of diabetes; estimates for year 2000 and projections for 2030. Diabetes Care 2004;21:1047-1053.

[3] Asia Pacific Cohort Studies Collaboration. The effects of diabetes on the risks of major cardiovascular diseases and death in the Asia-Pacific region. The Asia Pacific Cohort Studies Collaboration. Diabetes Care 2003;26:360-366.

[4] Huxley R, Barzi F, Woodward M. Excess risk of fatal coronary heart disease associated with diabetes in men and women: meta-analysis of 37 prospective cohort studies. BMJ 2006;332:73-78. 
[5] Ostgren CJ, Merlo J, Ra ${ }^{\circ}$ stam L, Lindblad U. Atrial fibrillation and its association with type 2 diabetes and hypertension in a Swedish community.Diabetes Obes Metab 2004;6:367-374.

[6] Kannel WB, Abbott RD, Savage DD, McNamara PM. Epidemiologic features of chronic atrial fibrillation: the Framingham study. N Engl J Med 1982;306: 1018-1022.

[7] Friberg J, Scharling H, Gadsbøll N, Truelsen T, Jensen GB, Copenhagen City Heart Study. Comparison of the impact of atrial fibrillation on the risk of stroke and cardiovascular death in women versus men (The Copenhagen City Heart Study). Am J Cardiol 2004;94:889-894

[8] Fuster V, Ryden LE, Cannom DS, et al. ACC/AHA/ESC 2006 guidelines for the management of patients with atrial fibrillation-executive summary: a report of the American College of Cardiology/American Heart Association Task Force on Practice Guidelines and the European Society of Cardiology Committee for Practice Guidelines (Writing Committee to Revise the 2001 Guidelines for the Management of Patients With Atrial Fibrillation). J Am Coll Cardiol 2006;48:854 -906 .

[9] Fuster V, Rydén LE, Cannom DS, Crijns HJ, Curtis AB, Ellenbogen KA, Halperin JL, Kay GN, Le Huezey JY, Lowe JE, Olsson SB, Prystowsky EN, Tamargo JL, Wann LS. 2011 ACCF/AHA/HRS Focused Updates Incorporated Into the ACC/AHA/ESC 2006 Guidelines for the Management of Patients with Atrial Fibrillation: a report of the American College of Cardiology Foundation/American Heart Association Task Force on Practice Guidelines Developed in partnership with the European Society of Cardiology and in collaboration with the European Heart Rhythm Association and the Heart Rhythm Society. J Am Coll Cardiol. 2011;57:e101-98

[10] Kerr CR, Humphries KH, Talajic M, Klein GJ, Connolly SJ, Green M, Boone J, Sheldon R, Dorian P, Newman D. Progression to chronic atrial fibrillation after the initial diagnosis of paroxysmal atrial fibrillation: results from the Canadian Registry of Atrial Fibrillation. Am Heart J. 2005;149:489-96.

[11] Nieuwlaat R, Prins MH, Le Heuzey JY, Vardas PE, Aliot E, Santini M, Cobbe SM, Widdershoven JW, Baur LH, Lévy S, Crijns HJ. Prognosis, disease progression, and treatment of atrial fibrillation patients during 1 year: follow-up of the Euro Heart Survey on atrial fibrillation. Eur Heart J. 2008;29:1181-9.

[12] De Vos CB, Pisters R, Nieuwlaat R, Prins MH, Tieleman RG, Coelen RJ, van den Heijkant AC, Allessie MA, Crijns HJ. Progression from paroxysmal to persistent atrial fibrillation clinical correlates and prognosis. J Am Coll Cardiol. 2010;55:72531.

[13] Nieuwlaat R, Capucci A, Camm AJ, Olsson SB, Andresen D, Davies DW, Cobbe S, Breithardt G, Le Heuzey JY, Prins MH, Lévy S, Crijns HJ; Atrial fibrillation management: a prospective survey in ESC member countries: the Euro Heart Survey on Atrial Fibrillation. Eur Heart J. 2005;26:2422-34.

[14] Pappone C, Radinovic A, Manguso F, Vicedomini G, Ciconte G, Sacchi S, Mazzone P, Paglino G, Gulletta S, Sala S, Santinelli V. Atrial fibrillation progression and management: A 5-year prospective follow-up study. Heart Rhythm 2008;5:1501-7. 
[15] Du X, Ninomiya T, de Galan B, Abadir E, Chalmers J, Pillai A, Woodward M, Cooper M, Harrap S, Hamet P, Poulter N, Lip GY, Patel A. Risks of cardiovascular events and effects of routine blood pressure lowering among patients with type 2 diabetes and atrial fibrillation: results of the ADVANCE study. Eur Heart J. 2009;30:1128-35. 


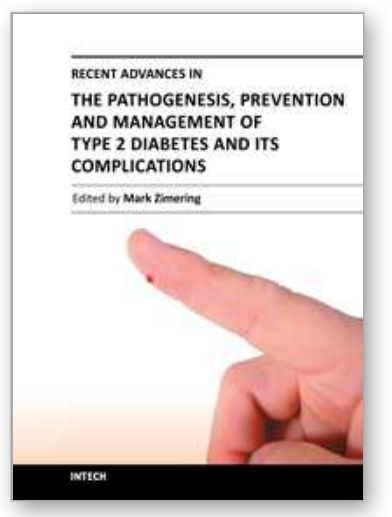

\author{
Recent Advances in the Pathogenesis, Prevention and \\ Management of Type 2 Diabetes and its Complications \\ Edited by Prof. Mark Zimering
}

ISBN 978-953-307-597-6

Hard cover, 442 pages

Publisher InTech

Published online 29, August, 2011

Published in print edition August, 2011

Type 2 diabetes â€œmellitusâ€ affects nearly 120 million persons worldwide- and according to the World Health Organization this number is expected to double by the year 2030. Owing to a rapidly increasing disease prevalence, the medical, social and economic burdens associated with the microvascular and macrovascular complications of type 2 diabetes are likely to increase dramatically in the coming decades. In this volume, leading contributors to the field review the pathogenesis, treatment and management of type 2 diabetes and its complications. They provide invaluable insight and share their discoveries about potentially important new techniques for the diagnosis, treatment and prevention of diabetic complications.

\title{
How to reference
}

In order to correctly reference this scholarly work, feel free to copy and paste the following:

Carlo Pappone, Francesca Zuffada and Vincenzo Santinelli (2011). AF and Diabetes Prognosis and Predictors, Recent Advances in the Pathogenesis, Prevention and Management of Type 2 Diabetes and its Complications, Prof. Mark Zimering (Ed.), ISBN: 978-953-307-597-6, InTech, Available from:

http://www.intechopen.com/books/recent-advances-in-the-pathogenesis-prevention-and-management-of-type2-diabetes-and-its-complications/af-and-diabetes-prognosis-and-predictors

\section{INTECH}

open science | open minds

\section{InTech Europe}

University Campus STeP Ri

Slavka Krautzeka 83/A

51000 Rijeka, Croatia

Phone: +385 (51) 770447

Fax: +385 (51) 686166

www.intechopen.com

\section{InTech China}

Unit 405, Office Block, Hotel Equatorial Shanghai

No.65, Yan An Road (West), Shanghai, 200040, China

中国上海市延安西路65号上海国际贵都大饭店办公楼 405 单元

Phone: +86-21-62489820

Fax: $+86-21-62489821$ 
(C) 2011 The Author(s). Licensee IntechOpen. This chapter is distributed under the terms of the Creative Commons Attribution-NonCommercialShareAlike-3.0 License, which permits use, distribution and reproduction for non-commercial purposes, provided the original is properly cited and derivative works building on this content are distributed under the same license. 